\title{
DEPOSITION OF YBCO THIN FILMS ON SILVER SUBSTRATE VIA A FLUORINE-FREE SOL-GEL SYNTHESIS
}

\author{
${ }^{1}$ Yongli Xu, ${ }^{1}$ Donglu Shi, ${ }^{2}$ L. Lian, ${ }^{2}$ M. Wang, ${ }^{1}$ Shaun M. McClellan \\ ${ }^{1}$ Department of Materials Science \& Engineering \\ University of Cincinnati \\ Cincinnati, OH 45220, USA \\ ${ }^{2}$ Dept. of Nuclear Engineering and Radiological Science \\ University of Michigan \\ Ann Arbor, MI 48109, USA
}

\begin{abstract}
To further develop grain-textured YBCO thin films for conductor development, we deposited, via a fluorine-free sol-gel synthesis, YBCO thin films on non-textured silver substrate. The interface structures were studied by both $\mathrm{X}$-ray diffraction (XRD) and transmission electron microscopy (HRTEM). XRD data indicated that the YBCO films on silver substrate exhibited $c$-axis grain orientations. Experimental details are reported on the sol-gel synthesis chemistry and XRD and HRTEM characterization of the YBCO thin films.
\end{abstract}

\section{INTRODUCTION}

In some large-scale applications of superconductors, the conductors are required not only to carry high critical current density at respectable magnetic fields, but also to possess high ductility, mechanical strength, and stability. To improve the transport properties of high-temperature superconductors, extensive experimental investigations have been carried out in developing highly textured thin films on a variety of substrates. ${ }^{1-5}$ The fabrication of $\mathrm{YBa}_{2} \mathrm{Cu}_{3} \mathrm{Ox}$ (YBCO) films by physical deposition on rolling assisted biaxially textured substrates (RABiTS) has proved to be successful in the conductor development for Labscale applications. ${ }^{1,2}$ The RABiTS technique uses well-established, possible scaleable, thermochamical processes to impart a high degree of grain texture to a base metal. Buffer layers are then deposited to yield chemically and structurally compatible surfaces. Epitaxial YBCO films are grown on such a surface, resulting in a critical current density at

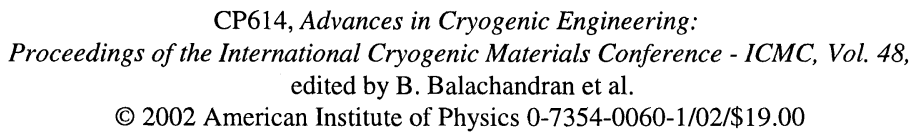


$77 \mathrm{~K}$ on the order of $10^{6} \mathrm{~A} / \mathrm{cm}^{2}{ }^{2,2}$. This approach has shown a promise for developing long conductors for industrial applications.

In our previous work $(4,5)$, we have shown the possibilities of obtaining $c$-axis grain orientated YBCO thick films on silver alloy substrates via high-temperature melt processing. Such a thick-film approach may serve as an alternative to the vapor deposition methods. The advantages of this approach includes: 1) YBCO is in direct contact with the metallic substrate that serves as a stabilizer; 2) it is readily scaled up into long-length conductors; 3 ) the large cross-sectional area of the thick film can potentially carry high engineering $J_{\mathrm{c}}$ 's, 4) no buffer layer is needed, so that the processing is straightforward and simple, and 5) the cost is much cheaper than for the vapor deposition methods.

Based on the knowledge and experimental data from the deposition of YBCO thick films on metallic substrates, we attempted to develop another viable approach to fabricating coated conductors. We selected a non-fluorine sol-gel method because of the difficulties with HF evolution from F-containing systems. ${ }^{6-14}$ To study the sol-gel chemistry of YBCO, in this work, we deposited the YBCO films on silver substrates. We present the experimental results of sol-gel synthesis and characterization of the YBCO thin films.

\section{EXPERIMENTAL DETAILS}

\section{Sample Preparation}

There have been extensive reports on fluorine-based sol-gel processes for the deposition of YBCO films. ${ }^{6-14}$ Our non-fluorine-based sol-gel YBCO solutions were developed in-house. For the precursor solution, stoichiometric (1:2:3) yttrium trimethylacetate, barium hydroxide, and copper trimethylacetate powders were dissolved in a mixed propionic acid/amine solvent with an oxide concentration between 0.1 and 0.5 $\mathrm{mol} / \mathrm{L}$. The addition of amine was important because it greatly improved the solubility of the precursor powders in propionic acid. The stock solution was stable in air with a shelf life longer than 2 years. Xylenes of alcohols were used for dilution and for controlling solution viscosity at $10-100 \mathrm{cP}$. The silver substrates used were commercial untextured, polycrystalline, high purity silver sheet of $0.5 \mathrm{~mm}$ in thickness. The substrates were cut from the silver sheets into typically $15 \times 15 \times 3 \mathrm{~mm}^{3}$ in dimension, and their top surfaces were mirror-polished down to $1 / 4 \mu \mathrm{m}$ with diamond paste. The films on silver substrates were deposited by spin coating at $3000-3500 \mathrm{rpm}$ and were dried at $200-250^{\circ} \mathrm{C}$ for several minutes. This process was repeated to build up the desired film thickness $(0.5-0.6 \mu \mathrm{m})$. The films were heated at $800-920^{\circ} \mathrm{C}$ under controlled atmosphere and $\mathrm{O}_{2}$ annealed at $400^{\circ} \mathrm{C}$ for 24 hours.

A Philips X-ray diffractometer with $\mathrm{Cu}-\mathrm{K} \alpha$ radiation was used to carry out the texture investigations. The $\mathrm{x}$-ray wavelength was $1.54 \AA$ with a beam size of $3 \mathrm{~mm}$. Scanning electron microscopy (SEM) experiments were performed on a Hitachi 2000. Some specimens were also examined by scanning electron microscopy in a JEOL JSM 5400 microscope that was operated at $10 \mathrm{kV}$. No conductive coating was applied. The highresolution TEM (HRTEM) experiments were performed on a JEOL JEM 4000EX TEM. Transport resistivity measurements were carried out down to liquid-helium temperature using a standard four-probe method.

\section{RESULTS AND DISCUSSIONS}

The YBCO sol-gel solutions were first applied to both yttrium-stabilized zirconia (YSZ), $\mathrm{LaAlO}_{3}$ (LAO) substrates via spin coating. In our previous studies we showed that 
these sol-gel films exhibited epitaxial growth confirmed by XRD and TEM.,14 Using the same film syntheses and heat-treatment procedures described in the experimental details, we deposited the YBCO films on untextured silver substrates. The x-ray diffraction (XRD) analysis of the film on the silver substrate is shown in Fig. 1. As can be seen in Fig. $1 \mathrm{a}$, all of the (001) peaks of the YBCO on silver exhibit strong intensities indicating a welltextured, $c$-axis oriented grain structure. A similar XRD pattern can be seen in Fig. 1b for the YBCO film on LAO as a comparison. These are quite typical XRD patterns observed from most of sol-gel films on YSZ and LAO in our experiments. In Fig. 2 the SEM images show that the YBCO film appears to have a multi-grain structure, but is quite dense with sharp irregular edges rather than the typical platelets (Fig. 2a). The interface also appears to be smooth without any reactions (Fig. 2b).

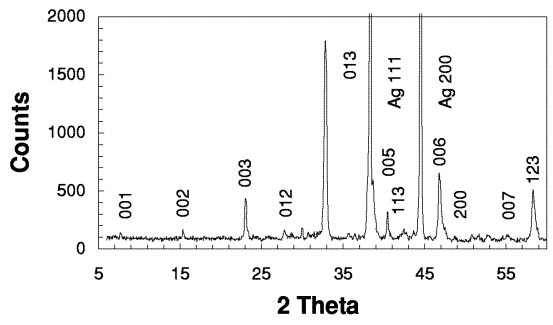

Figure 1a. XRD pattern for YBCO film on silver substrate

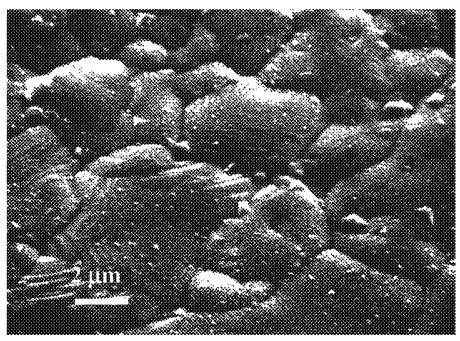

Figure 2a. SEM microphotograph showing the surface of the YBCO film on silver



Figure 1b. XRD pattern for YBCO film on LAO substrate

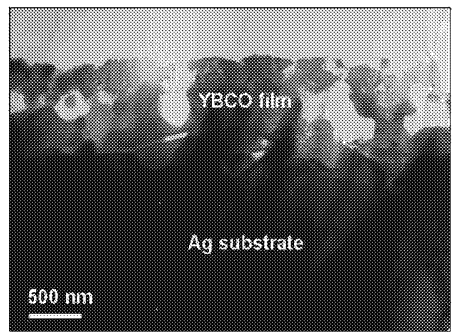

Figure 2b. SEM micro-photograph showing the cross sectional area of YBCO film on silver

Figure 3a shows the TEM image of the interface between the YBCO film and the silver substrate. The interface is clean with coherent structural characteristics. However, we also observed precipitates within the YBCO phase as shown in Fig. 3b. The precipitate has caused severe lattice distortion as can be seen from the image in Fig. $3 \mathrm{~b}$.

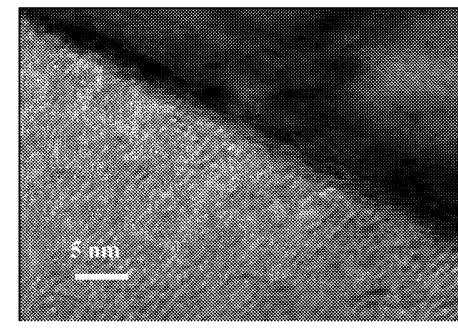

Figure 3a. TEM image of YBCO/silver Interface

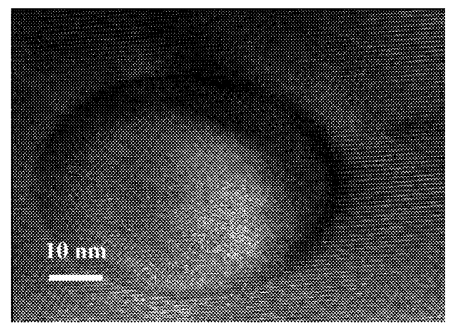

Figure 3b. HRTEM showing the precipitate within the YBCO phase

The interface morphology and structure were further studied by using EDX, which 
enabled us to see the variation in stoichiometry near the interface area. Figure 4a shows the TEM image in which several $\mathrm{Ba}-$ and $\mathrm{Cu}$ - rich areas were identified. From this result one can conclude that chemical segregation occurred at the interface area. The morphology at the interface indicated saw-tooth-like interface as shown in Figure 4b. Such morphology may strongly influence the initial grain growth and orientation. As a result of the soft nature of silver, unlike those of LAO and YSZ, it is difficult to obtain a smooth surface via polishing. This rough surface of the substrate has lead to a high roughness at the interface. However, further growth of the film appears to result in an improved grain orientation at the top surface of the film as shown in Figure 1a. This enhanced texture could be due to the platelet YBCO grain morphology as they tend to line up to reduce the total system energy.

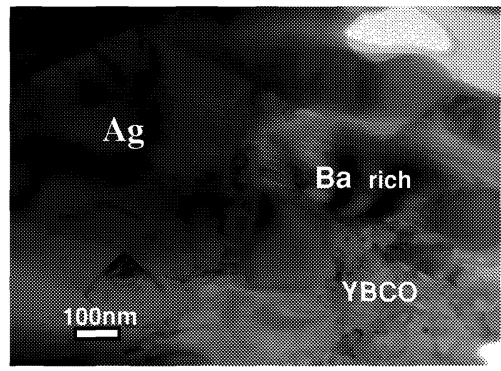

Figure 4a. TEM image showing the Baand $\mathrm{Cu}$ - rich regions near the interface

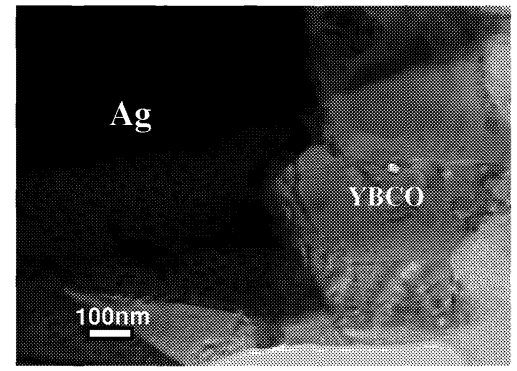

Figure 4b. TEM image showing the interface Morphology

The electrical resistivity of the YBCO film on silver substrate is shown in Figure 5. As can be seen, the resistivity exhibits a typical metallic behavior in the normal state with a decreasing slope. However, the transition is quite broad and it does not fall to a zeroresistance state down to $77 \mathrm{~K}$. This is likely due to silver diffusion at the YBCO grain boundaries. Since the heat treatment temperature $810^{\circ} \mathrm{C}$ is quite high, the silver diffusion is assumed to be very active and can easily penetrate the $0.5 \mu \mathrm{m}$ thick YBCO film. As the grain boundaries of YBCO are coated by the silver, the resistivity remains finite even at low temperatures. One of the solutions to this problem would have to be the heat treatment at relative lower temperatures so that the silver diffusion is reduced. For comparison, we also show the $\mathrm{T}_{\mathrm{c}}$ result for an YBCO film on LAO as shown in Fig. 6 . This indicates that the non-fluorine sol-gel method has resulted in a high-quality film.

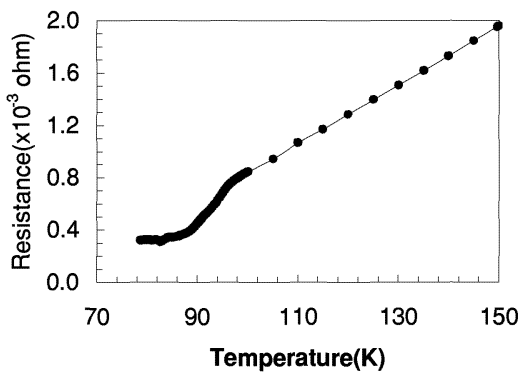

Figure 5. Resistance versus temperature for the YBCO film on silver substrate



Figure 6. Resistivity versus temperature for the YBCO film on LAO substrate 


\section{CONCLUSIONS}

YBCO thin films have been deposited on silver substrate via a non-fluorine sol-gel synthesis. Both XRD and electron microscopy results have shown that the YBCO film exhibits considerable grain texture and no adverse reaction has been found at the interface area. However, chemical segregation occurred near the interface resulting in $\mathrm{Cu}$ - and $\mathrm{Ba}-$ rich regions. It is also found that the electrical resistivity does not fall to the zeroresistance state, possibly due to severe silver diffusion into the YBCO grain boundaries.

\section{ACKNOWLEDGMENT}

This work was supported by a grant from National Science Foundation under contract number ECS-9802281.

\section{REFERENCES}

1. A. Goyal et al., Appl. Supercon., 4, 403-427, (1996).

2. M. Paranthaman et al., Proc. of the 9th Int. Symposium on Supercond, Sapporo, Hokkaido, Japan, Oct. 21-24, 1996.

3. X. D. Wu et al., Appl. Phys. Lett., 65, 1961, (1994).

4. D. Shi, D. Qu, X. Wen, B. A. Tent, and M.Tomsic, J. Superconductivity, 11, 575, (1998)

5. D. Shi, Yongli Xu, S. X. Wang, L. M. Wang, and Shaun M. McClellan, Physica C, 353, 258-264 (2001).

6. M. E. Gross, M. Hong, S. H. Liou, P. K. Gallagher, and J. Kwo, Appl. Phys. Lett. 52, 160-162, (1988).

7. M. L. Kullberg, M. T. Lanagan, W. Wu, and R. B. Poeppel, Supercond. Sci. Technol. 4, 337-342, (1989).

8. Y. L. Chen, J. V. Mantese, A. H. Hamdi, and A. L. Mecheli, J. Mater. Res. 4,1065-1071, (1989).

9. K. Yamagiwa, H. Hiei, Y. Takahashi, S. B. Kim, K. Matsumoto, H. Ikuta, U. Mizutani, and I. Hirabayashi, Physica C, 334, 301-305, (2000).

10. A. Gupta, R. Jagannathan, E. I. Cooper, E. A. Geiss, J. I. Landmanm and B.W. Hussey, Appl. Phys. 71, 1868-1877, (1992).

11. P. C. McIntyre, M. J. Cima, J. A. Smith Jr., R. B. Hallock, M. P. Siegal, and J. M. Phillips, J. Appl. Phys. 71,1868-1877, (1992).

12. P. C. Mcintyrem M. J. Cima, and A. Roshko, J. Appl. Phys. 77, 5263-5272, (1995)

13. A. Malozemoff, S. Annavarapu, L. Fritzmeier, Q. Li, V. Prunier, M. Rupich, C. Thieme, W. Zhang, A. Goyal, M. Paranthaman, and D. F. Lee, Supercond. Sci. Technol. 13, 473-476, (2000)

14. D. Shi, Yongli Xu, L. Lian, S. X. Wang, L. M. Wang, Shaun M. McClellan, R. Buchanan, and K. C. Goretta, submitted to Physica C, 2001. 